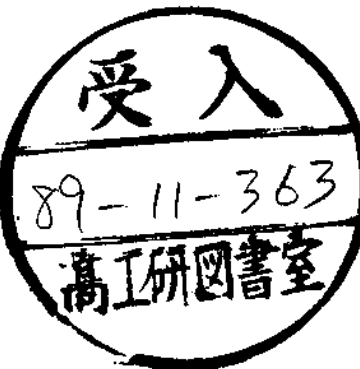

\title{
The Superconducting Super Collider
}

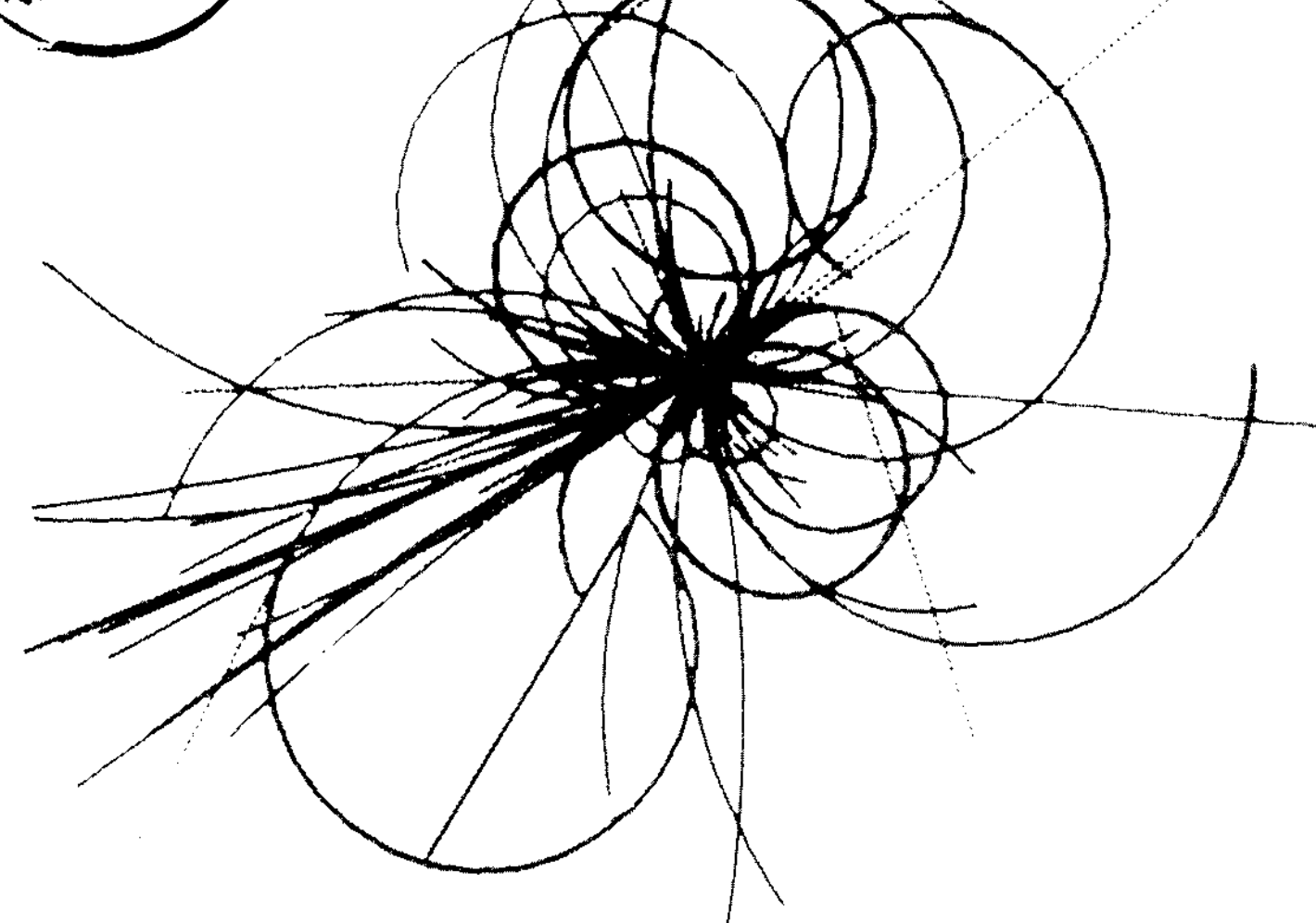

Diffusive Losses From SSC Particle Bunches Due to Long-Range Beam-Beam Interactions

John Irwin

SSC Central Design Group

September 1989 


\title{
Diffusive Losses from SSC Particle Bunches \\ Due to Long-Range Beam-Beam Interactions
}

\author{
John Irwin* \\ SSC Central Design Group ${ }^{\dagger}$ \\ Lawrence Berkeley Laboratory, 90-4040 \\ Berkeley, California 94720
}

September 1989

* Former address: Wilson Laboratory, Cornell University, Ithaca, NY 14853.

$\dagger$ Operated by Universities Research Association, Inc. for the U.S. Department of Energy. 


\begin{abstract}
A B S TRACT
The long-term motion of particles in the tails of SSC particle bunches has been simulated, including effects of the short- and long-range beam-beam interactions, the nonlinear forces from the interaction region quadrupoles, and tune modulation. The author shows that a "diffusive dynamic aperture" can be well defined, and then determines its value for a range of machine parameters and tune modulations. A simple picture emerges indicating that the $1 / r$ force of the long-range beam-beam interaction drives the observed diffusion. The simulations indicate that the proposed crossing angle of $75 \mu \mathrm{rad}$ at the low$\beta^{*} \mathrm{IPS}$ is adequate, but increase of beam intensity would require a larger crossing angle.
\end{abstract}

\title{
TABLE OF CONTENTS
}

1. INTRODUCTION

Task

General Method

2. SiMULATION METHODS

Short-Range Beam-Beam Force

Long-Range Beam-Beam Force

Nonlinear Triplet Quadrupoles

Tune Modulation

3. FEW-TURN RESULTS

Turn-by-Tum Variance

Multi-Turn Averages

Tune Plane Considerations

4. MANY-TURN RESULTS

Diffusive Boundary With and Without Tune Modulation

Change in Diffusive Boundaries as Parameters are Varied

5. CONCLUSIONS

6. ACKNOWLEDGEMENTS 


\section{INTRODUCTION}

\subsection{TASK}

An important design parameter for the SSC is the crossing angle of the two beams at the interaction points. The smaller this angle, the stronger the interaction between bunches in the two beams as they approach the interaction point (IP) and the more likely that particles may be lost from the beam. On the other hand, the larger this angle the smaller the machine luminosity and the larger the nonlinear forces in the interaction region (IR) triplet quadrupoles.

A general rule has evolved from experience with proton machines known as the "7 $\sigma$ rule," which asserts that bunches separated by $7 \sigma$ will not significantly disturb one another. Conforming to this rule, the crossing angle of $75 \mu \mathrm{rad}$ at the $\beta=0.5$ IPs of the SSC[1] implies that bunches will be separated by $7.5 \sigma$ as they approach and depart the IP. However, in each IR there are sixty long-range interactions that have the same magnitude and occur with approximately the same phase. Furthermore, they occur in combination with a nonlinear force at the IP, which is referred to as the short-range beam-beam force, and significant nonlinear forces in the IR triplet quadrupoles.

It is the purpose of this investigation to simulate the long-range beam-beam force in combination with the short-range beam-beam force, the nonlinearities from the triplet quadrupoles and a modulation of machine tune, and to observe any indication of particle loss from the bunches, thereby examining the choice of $75 \mu \mathrm{rad}$ for the crossing angle. Only single particle effects are studied; coherent long-range beam-beam effects have been examined elsewhere. [2]

\subsection{GENERAL METHOD}

A straightforward simulation of particle loss might proceed by tracking an adequate sample of particles, perhaps 1000 , for a number of revolutions approaching the design storage time of the SSC beam (about $3 \times 10^{8}$ ) for tens of initial machine parameters and conditions. Roughly, it would be necessary to simulate $10^{11}$ particle-turns. Experience with fast codes on available computers indicates one might hope to track $10^{7}$ particle turns in a few minutes of CPU time, suggesting that this approach would require more than two months of dedicated CPU time on a machine like the CRAY- 2 .

A workable alternative is to start samples of 100 particles at select transverse amplitudes with randomized phases, since amplitudes are constants of the motion for linear machines and are slowly varying quantities under the influence of small nonlinear 
perturbations. The samples are observed for $10^{6}$ turns, long enough to detect the presence of any diffusion away from the initial amplitude. Since one expects that diffusion will increase with increasing amplitude, investigation of a set of ten amplitudes can establish potential for particle loss. This requires about $10^{9}$ particle turns, or about 100 minutes of CPU time. The total project, involving tens of initial machine conditions, can be accomplished with a couple thousand minutes of CPU time.

The presence of diffusion is evident as a steady increase in the spread of the initial distribution, best measured quantitatively by the statistical quantity known as the variance (VAR), the mean value of the second moment of the distribution. In a pure diffusive situation one expects

$$
V A R=V_{0}+\alpha N_{T},
$$

where $N_{T}$ is the number of turns, and $\alpha$ is some proportionality constant depending on the strength of the diffusion.

For purposes of comparing different machine conditions we define a "diffusive dynamic aperture" (DDA), determined by a specification on the rate of growth of the variance. We choose to define the boundary of this aperture as a diffusive spread in amplitude equal to the original core radius in $10^{8}$ turns (eight hours for the SSC). This amounts to an increase of the variance by an amount equal to the initial beam emittance, $\epsilon$, in eight hours. Using the linearity condition on diffusive variance growth, the DDA boundary could equivalently be defined by a variance growth of $0.01 \epsilon$ in $10^{6}$ revolutions; this is the definition I use here. It came to my attention when this paper was in proof that this idea was proposed by A. Dragt in 1986.[3]

\section{Simulation MODEL}

\subsection{THE SHORT-RANGE BEAM-BEAM FORCE}

The SSC is designed to have round beams at the IP; the horizontal and vertical emittances are equal, and the horizontal and vertical $\beta$ functions are equal at the IP. Furthermore, since the longitudinal dimension is much greater than the transverse dimension, the geometry is cylindrical, and it is possible to calculate the electric and magnetic fields for any beam profile. 
In particular, if we assume that the beam profile is Gaussian, the change in the $x$-component of the slope of a particle passing through the bunch at coordinates $(x, y)$ is given by

$$
\frac{\delta x^{\prime}}{\sigma_{x^{\prime}}^{*}}=\frac{\epsilon_{N 0}}{\epsilon_{N}} \frac{x}{\sigma_{x}^{*}} \frac{1}{R^{* 2}}\left[1-\exp \left(-R^{* 2}\right)\right]
$$

where $\sigma_{x}^{*}=\sqrt{\epsilon \beta_{x}^{*},} \sigma_{x^{\prime}}^{*}=\sqrt{\epsilon / \beta_{x}^{*},}, R^{* 2}=\left(x^{2}+y^{2}\right) / 2 \sigma_{x}^{* 2}, \epsilon=\epsilon N^{\prime} \gamma, \epsilon N \approx 1.0 \times 10^{-6}$ mrad is the normalized emittance, and $\epsilon_{N 0}=N_{B} r_{p} . N_{B} \approx 7.3 \times 10^{9}$ is the number of particles per bunch, $r_{p}$ is the classical radius of the proton, and $\gamma$ is the Lorentz factor at 20 $\mathrm{TeV}$. The ratio $\epsilon_{N 0} \epsilon_{N}(\approx 0.011)$ is equal to twice the beam-beam phase shift (= the beam-beam tune shift $\times 4 \pi)$.

Equation (2.1) assumes head-on crossing. See Tennyson for corrections due to nonzero crossing angles. ${ }^{[4]}$

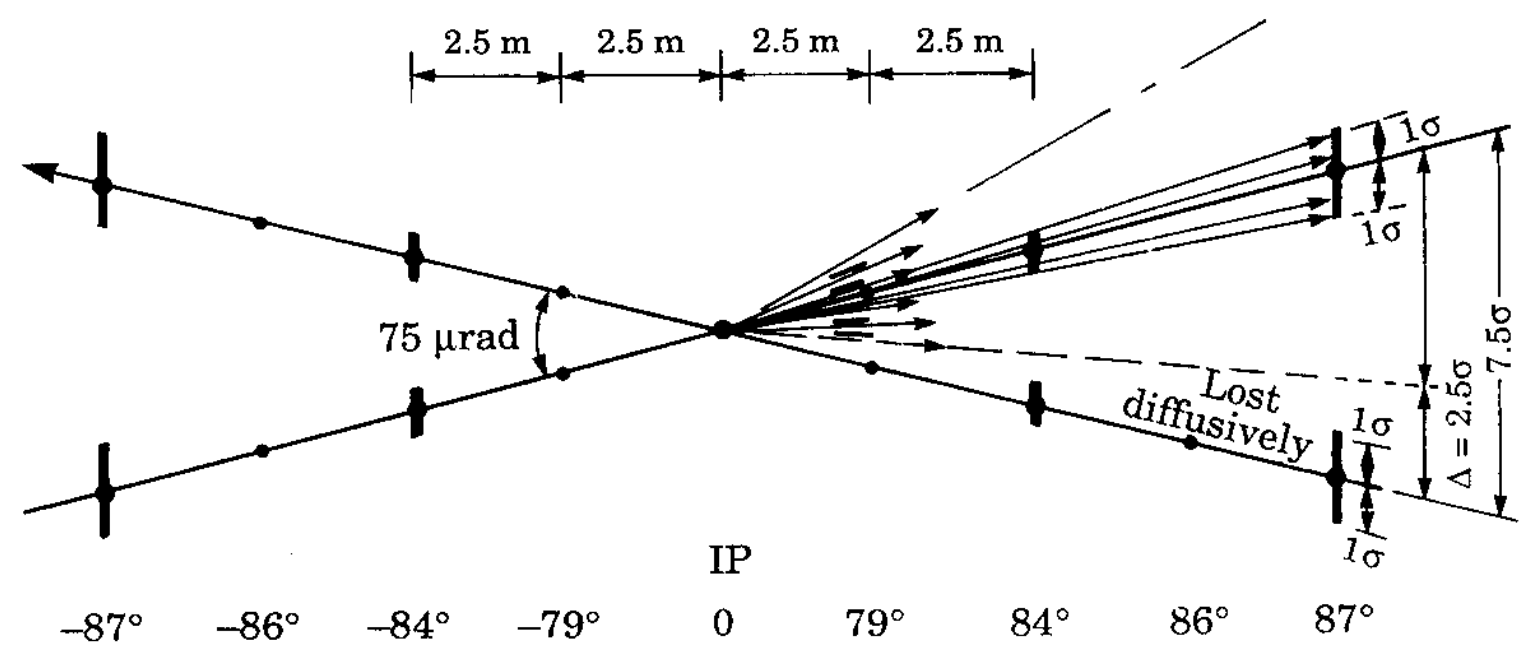

Betatron Phase Relative to IP Phase

Figure 1. One of the two low- $\beta$ IPs showing eight of sixty LR interaction locations. Approaching and departing bunches pass counter-rotating bunches every $2.5 \mathrm{~m}$. The betatron phase for all encounters is close to either $\pm 90^{\circ}$ from the phase at the IP. We find that, given the conditions described in Table 2 , particles within $\Delta \approx 2.5 \sigma$ of the counterrotating beam are lost diffusively. 


\subsection{THE LONG-RANGE BEAM-BEAM FORCE}

Particle bunches pass one another about every $2.5 \mathrm{~m}$ as they approach and depart the IP. Approximately eight interactions occur in the field-free region on each side of the IP, and 22 interactions occur within each IR triplet quadrupole.

If in a fixed lab coordinate system the slope of the center line of the beam bunch at the IP has coordinates $\left(d_{x}^{\prime} / 2, d_{y}^{\prime} / 2\right)$ and if the coordinates of the center of the bunch at the $i^{\text {th }}$ IP are $\left(d_{x}^{i} / 2, d_{y}^{i} / 2\right)$ and the center of the counter-rotating beam is $\left(-d_{x}^{i} / 2,-d_{y}^{i} / 2\right)$, then in the field-free region where the horizontal and vertical $\beta$ functions are equal, the $x$-component of the kick given to a particle passing at coordinates $\left(x^{i}, y^{i}\right)$ (measured from the centerline of the beam) is given by

$$
\frac{\delta x^{i^{\prime}}}{\sigma_{x^{\prime}}^{i}}=\frac{\epsilon_{N 0}}{\epsilon_{N}} \frac{x^{i}+d_{x}^{i}}{\sigma_{x}^{i}} \frac{1}{R_{d}^{i 2}}\left[1-\exp \left(-R_{d}^{i 2}\right)\right]
$$

Here $\sigma_{x}^{i}=\sqrt{\epsilon \beta_{x}^{i}}, \sigma_{x}^{i}=\sqrt{\epsilon / \beta_{x}^{i}}$ and $R_{d}^{i 2}=\left[\left(x^{i}+d_{x}^{i}\right)^{2}+\left(y^{i}+d_{y}^{i}\right)^{2}\right] / 2 \sigma_{x}^{i 2}$.

The phase advance from the IP to the $i^{\text {th }}$ interaction is given by

$$
\Delta \phi^{i}=\int_{s=0}^{s=s^{i}} \frac{d s}{\beta(s)} .
$$

Since in the field-free region $\beta(s)=\beta^{*}+s^{2} / \beta^{*}$, the above integral is an inverse tangent function. Assuming that $\beta^{*}=0.5 \mathrm{~m}$ and that long-range interactions occur every $2.5 \mathrm{~m}$, we find $\Delta \phi^{i}=\left\{79^{\circ}, 84^{\circ}, 86^{\circ}, 87^{\circ}, 88^{\circ}, \ldots, 88.6^{\circ}\right\}$ for the eight IPs in the field-free region.

If we assume that all these phase differences are equal to $90^{\circ}$, then in Eq. (2.2) we may set

$$
\frac{\delta x^{i^{\prime}}}{\sigma_{x^{\prime}}^{i}}=-\frac{\delta x}{\sigma_{x}^{*}}, \quad \frac{x^{i}+d_{x}^{i}}{\sigma_{x}^{i}}=\frac{x^{\prime}+d_{x}^{\prime}}{\sigma_{x^{\prime}}^{*}}, \quad R_{d}^{i 2}=R_{d^{\prime}}^{* 2}
$$

where $R_{d^{\prime}}^{* 2}=\left[\left(x^{\prime}+d_{x}^{\prime}\right)^{2}+\left(y^{\prime}+d_{y}^{\prime}\right)^{2}\right] / 2 \sigma_{x^{\prime}}^{*}$. This result demonstrates that the calculations can be referred simply to the coordinates at the IP, and all kicks from the field free space are identical. At a horizontal crossing, specifications call for $d_{x}^{\prime}=7.5 \sigma_{x^{\prime}}^{*}$ and $d_{y}^{\prime}=0$.

The phase change $\Delta \phi^{i} \approx-90^{\circ}$ for the interactions that occur before the IP. For these interactions

$$
\frac{\delta x^{i^{\prime}}}{\sigma_{x}^{i}}=-\frac{\delta x}{\sigma_{x}^{*}}, \quad \frac{x^{i}+d_{x}^{i}}{\sigma_{x}^{i}}=-\frac{x^{\prime}+d_{x}^{\prime}}{\sigma_{x^{\prime}}^{*}}, \quad R_{d}^{i 2}=R_{d^{\prime}}^{* 2}
$$


Thus the kicks before and after the IP are identical. The resulting algorithm for the long-range beam-beam kicks is given by

$$
\frac{\delta x}{\sigma_{x}^{*}}=-N_{k} \frac{\epsilon_{N 0}}{\epsilon_{N}}\left[\frac{x^{\prime}+d_{x}^{\prime}}{\sigma_{x^{\prime}}^{*}} \frac{1}{R_{d^{\prime}}^{* 2}}\left[1-\exp \left(-R_{d^{\prime}}^{* 2}\right)\right]-\frac{d_{x}^{\prime}}{\sigma_{x^{\prime}}^{*}} \frac{1}{R_{d^{\prime}}^{02}}\left[1-\exp \left(-R_{d^{\prime}}^{02}\right)\right]\right],
$$

where $N_{k}$ is the total number of kicks and $R_{d}^{02}=\left(d_{x}^{2}+d_{y}^{\prime 2}\right) / 2 \sigma_{x^{\prime}}^{* 2}$. The second term in the brackets subtracts out the value of the kicks for $x^{\prime}=y^{\prime}=0$. In other words we are inserting a dipole kick to offset the dipole kick of the long-range beam-beam force.

Because the actual phase rotation is not $90^{\circ}$, there are small corrections to these formula. Letting $\delta=\left|\delta x / \sigma_{x}^{*}\right|$ be the magnitude of the kick, the corrections are the order of $\cos \left(\Delta \phi^{\dot{ }}\right) \delta$. However, there is a cancellation at each phase angle from the kicks before and after the IP. Correction terms are finally the order of $\cos ^{2}\left(\Delta \phi^{i}\right) \delta^{2}$. As a consequence, the $90^{\circ}$ approximation is very good.

Within the IR triplet quadrupoles the horizontal and vertical $\beta$ functions are unequal, and the cylindrical symmetry is broken. (See Figure 2 for a sketch of the $\beta$ functions.) For half the length of the triplet quad the $\beta$ functions are within a factor of two of one another, hence the square root of their ratio, a measure of the distortion of the counterrotating beam, is less than 1.4. Since this ratio is sometimes greater and sometimes less than 1.0 , one might expect some cancellation. The distortion ratio is greater than 2.0 for only $10 \mathrm{~m}$, and then is always less than 2.4 . Since the important region for our simulations is greater than 2.5 core radii from the counter-rotating beam I believe it is adequate, for purposes of estimating the long-range beam-beam interaction in the triplet quadrupoles, to assume the $\beta$ functions are equal there. Following the arguments presented above, the magnitude of the kicks within the triplet quads are identical to the kicks from the field-free region. Thus we may account for all the long-range beam-beam kicks by setting $N_{k}=60$ in Eq. (2.6). For perhaps ten of these kicks the departure from cylindrical symmetry is cause for concern. In the interest of establishing a base case with good computational speed I will simply use the cylindrical symmetric formulation.

Of course, even though all the kicks are approximately identical when referred to the coordinates at the IP, some details that result from small changes in particle position as the particle passes from interaction to interaction are neglected. For example, over the course of the 30 kicks that occur before the IP, the dipole term will shift the orbit position by about $0.1 \sigma_{x}^{*}$ 


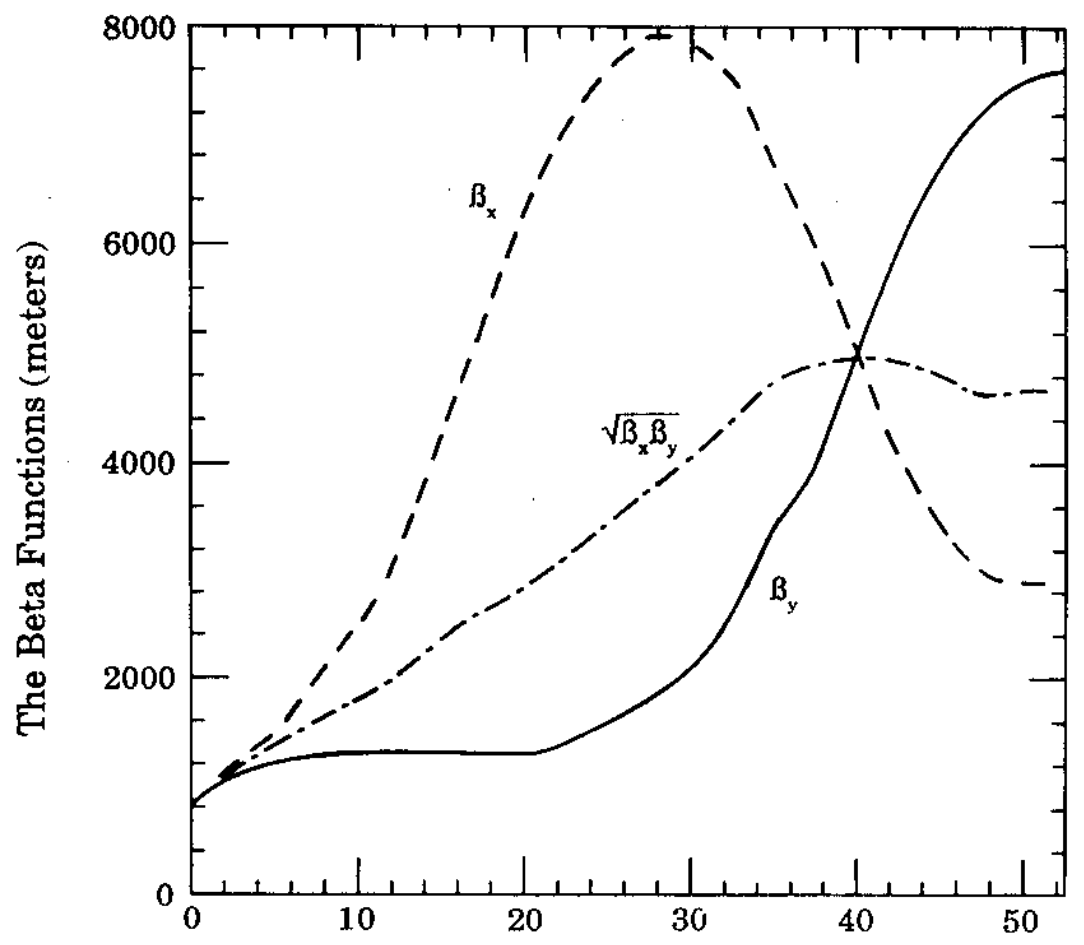

Position Within the Triplet Quad (meters)

Figure 2. The $\beta$ functions in the IR triplet quad.

\subsection{THE NONLINEAR TR IPLET QUADRUPOLE FORCES}

The longitudinally averaged magnetic field within the triplet quadrupoles is given by the expression

$$
B_{y}+i B_{x}=B_{0} \times 10^{-4} \sum\left(a_{n}+i b_{n}\right) 10^{2 n}(\hat{x}+i \hat{y})^{n}
$$

where $B_{0}$ is the average field within the dipole magnets, and $\hat{x}$ and $\hat{y}$, measured in meters, are the transverse displacements from the centerline of the magnets. The nonlinear contributions begin with the $n=2$ term in this sum. The design specifications for the rms values of these coefficients after correction are ${ }^{[4]}$

$$
\left\{b_{n}\right\}=\left\{a_{n}\right\}=\left\{a_{2}, \ldots, a_{9}\right\}=\{0.1,0.1,0.1,0.1,0.06,0.05,0.05,0.04\} .
$$

In subsequent formulas, $\tilde{a}_{n}=a_{n} 10^{2 n}$ and $\tilde{b}_{n}=b_{n} 10^{2 n}$.

Since $\Delta \phi^{j} \approx 90^{\circ}$ at all IPs within the triplet quadrupoles, it follows that the $\hat{x}$-coordinate of the center of the beam is $d_{x}(s) / 2=\sqrt{\beta_{x}^{*} \beta_{x}(s)} \mathrm{d}_{x}^{\prime} / 2$. Likewise, the particle coordinates within the quadrupole are given by expressions like

$$
\hat{x}(s)=x(s)+\frac{d_{x}(s)}{2}=\left(x^{\prime}+\frac{d_{x}^{\prime}}{2}\right) \sqrt{\beta_{x}^{*} \beta_{x}(s)} \equiv \hat{x}^{\prime} \sqrt{\beta_{x}^{*} \beta_{x}(s)} .
$$


Combining Eqs. (2.7) and (2.8) we find the following expressions for the kicks due to these multipoles:

$$
\begin{aligned}
& \delta x=-\frac{E_{R}}{E_{0}} \operatorname{Re}\left[\sum\left(\tilde{a}_{n}+i \tilde{b}_{n}\right) \beta^{*}(n+1) / 2 \int \sqrt{\beta_{x}(s)}\left(\hat{x}^{\prime} \sqrt{\beta_{x}(s)}+i \hat{y}^{\prime} \sqrt{\beta_{y}(s)}\right)^{n} \frac{d s}{L}\right], \\
& \delta y=+\frac{E_{R}}{E_{0}} \operatorname{Im}\left[\sum\left(\tilde{a}_{n}+i \tilde{b}_{n}\right) \beta^{*}(n+1) / 2 \int \sqrt{\beta_{y}(s)}\left(\hat{x}^{\prime} \sqrt{\beta_{x}(s)}+i \hat{y}^{\prime} \sqrt{\beta_{y}(s)}\right)^{n} \frac{d s}{L}\right],
\end{aligned}
$$

where $E_{R}=10^{-4} e c B_{0} L \approx 10 \mathrm{MeV}$ and $E_{0}=20 \mathrm{TeV}$.

As a first estimate, $\beta_{x}(s)$ and $\beta_{y}(s)$ are replaced by their geometric mean $\beta_{G}(s)$ $=\sqrt{\beta_{x}(s) \beta_{y}(s)}$. With this approximation

$$
\begin{aligned}
& \delta x=-\frac{E_{R}}{E_{0}} \operatorname{Re}\left[\sum\left(\tilde{a}_{n}+i \tilde{b}_{n}\right)\left(\hat{x}^{\prime}+i \hat{y}^{\prime}\right)^{n} \beta^{*(n+1) / 2} \int \beta_{G}(s)^{(n+1) / 2} \frac{d s}{L}\right] . \\
& \text { If } \bar{\beta}_{G}=\int \beta_{G}(s) \frac{d s}{L} \text { and } g_{n}^{\beta}=\int \beta_{G}(s)^{(n+1) / 2 \frac{d s}{L} / \bar{\beta}_{G}^{(n+1) / 2} \text { then }} \\
& \qquad \delta x=-\frac{E_{R}}{E_{0}} \operatorname{Re}\left[\sum\left(\tilde{a}_{n}+i \tilde{b}_{n}\right)\left(\hat{x}^{\prime}+i \hat{y}^{\prime}\right)^{n}\left(\beta^{*} \bar{\beta}_{G}\right)^{(n+1) / 2} g_{n}^{\beta}\right] .
\end{aligned}
$$

Using the $\beta$ functions given in Figure $2, \bar{\beta}_{G} \approx 3400 \mathrm{~m}$ and

$$
g_{n}^{\beta}=\{1.0,1.07,1.17,1.22,1.48,1.69,1.95,2.27,2.65\}
$$

To further explore the accuracy of this approximation, we expand the binomial expression in the integrand of Eqs. (2.9) and (2.10), obtaining integrals of the form

$$
\begin{aligned}
& B_{x}(n, k) \equiv \int \beta_{x}(s)^{(n+1-k) / 2} \beta_{y}(s)^{k / 2} \frac{d s}{L} \\
& B_{y}(n, k) \equiv \int \beta_{x}(s)^{(n-k) / 2} \beta_{y}(s)^{(k+1) / 2} \frac{d s}{L} .
\end{aligned}
$$

Table 1 gives the values of these integrals divided by the geometric mean approximation, for example

$$
b_{x}(n, k) \equiv B_{x}(n, k) / \int \beta_{G}(s)^{(n+1) / 2} \frac{d s}{L}
$$


The quantity $\bar{b}_{x}(n)$ in the table is the average of the $b_{x}(n, k)$ weighted by the appropriate binomial coefficients. Finally, we define $\frac{-}{g} \beta \equiv 0.5\left[b_{x}(n, k)+b_{y}(n, k)\right] g_{n}^{\beta}$ and replace $g_{n}^{\beta}$ by $\bar{g}_{n}^{\beta}$ when calculating $\delta x$ using Eq. (2.12).

$$
\bar{g}_{n}^{\beta}=\{1.07,1.17,1.32,1.51,1.74,2.03,2.38,2.81,3.30\} .
$$

Table 1. Moments of $\beta$ functions within triplet quadrupoles.

\begin{tabular}{|c|c|c|c|c|c|c|c|c|c|c|c|}
\hline$\underline{n}$ & $k$ & $b_{x}(n, k)$ & $\bar{b}_{x}(n)$ & $b_{\mathrm{y}}(n, k)$ & $\bar{b}_{y}(n)$ & $\underline{n}$ & $k$ & $b_{x}(n, k)$ & $\bar{b}_{x}(n)$ & $b_{y}(n, k)$ & $\bar{b}_{y}(n)$ \\
\hline 2 & $\begin{array}{l}0 \\
1 \\
2\end{array}$ & $\begin{array}{l}0.95 \\
0.94 \\
1.12\end{array}$ & 0.99 & $\begin{array}{l}1.00 \\
1.12 \\
1.63\end{array}$ & 1.20 & 7 & $\begin{array}{l}0 \\
1 \\
2\end{array}$ & $\begin{array}{l}2.24 \\
1.61 \\
1.22\end{array}$ & 1.16 & $\begin{array}{l}1.61 \\
1.22 \\
1.03\end{array}$ & 1.28 \\
\hline 3 & $\begin{array}{l}0 \\
1 \\
2 \\
3\end{array}$ & $\begin{array}{l}1.08 \\
0.95 \\
1.00 \\
1.29\end{array}$ & 1.03 & $\begin{array}{l}0.95 \\
1.00 \\
1.29 \\
1.98\end{array}$ & 1.22 & & $\begin{array}{l}3 \\
4 \\
5 \\
6\end{array}$ & $\begin{array}{l}1.00 \\
1.16 \\
1.60 \\
2.56\end{array}$ & & $\begin{array}{l}1.16 \\
1.60 \\
2.56 \\
4.50\end{array}$ & \\
\hline 4 & $\begin{array}{l}0 \\
1 \\
2 \\
3 \\
4\end{array}$ & $\begin{array}{l}1.27 \\
1.03 \\
0.96 \\
1.09 \\
1.50\end{array}$ & 1.06 & $\begin{array}{r}1.03 \\
.96 \\
1.09 \\
1.50 \\
2.41\end{array}$ & 1.24 & 8 & $\begin{array}{l}0 \\
1 \\
2 \\
3 \\
4 \\
5\end{array}$ & $\begin{array}{l}2.73 \\
1.92 \\
1.42 \\
1.13 \\
1.00\end{array}$ & 1.19 & $\begin{array}{l}1.92 \\
1.42 \\
1.13 \\
1.00 \\
1.04\end{array}$ & 1.28 \\
\hline 5 & $\begin{array}{l}0 \\
1 \\
2\end{array}$ & $\begin{array}{l}1.51 \\
1.16 \\
1.00\end{array}$ & 1.10 & $\begin{array}{l}1.16 \\
1.00 \\
1.00\end{array}$ & 1.25 & & $\begin{array}{l}5 \\
6 \\
7\end{array}$ & $\begin{array}{l}1.04 \\
1.29 \\
1.89\end{array}$ & & $\begin{array}{l}1.29 \\
1.89 \\
3.11\end{array}$ & \\
\hline & $\begin{array}{l}3 \\
4 \\
5\end{array}$ & $\begin{array}{l}1.00 \\
1.21 \\
1.77\end{array}$ & & $\begin{array}{l}1.22 \\
1.77 \\
2.95\end{array}$ & & 9 & $\begin{array}{l}0 \\
1 \\
2 \\
3\end{array}$ & $\begin{array}{l}3.35 \\
2.31 \\
1.67 \\
1.27\end{array}$ & 1.21 & $\begin{array}{l}2.32 \\
1.67 \\
1.27\end{array}$ & 1.30 \\
\hline 6 & $\begin{array}{l}0 \\
1 \\
2 \\
3 \\
4 \\
5 \\
6\end{array}$ & $\begin{array}{l}1.84 \\
1.35 \\
1.08 \\
1.00 \\
1.06 \\
1.38 \\
2.12\end{array}$ & 1.13 & $\begin{array}{l}1.35 \\
1.08 \\
1.00 \\
1.06 \\
1.38 \\
2.12 \\
3.64\end{array}$ & 1.27 & & $\begin{array}{l}5 \\
4 \\
5 \\
6 \\
7\end{array}$ & $\begin{array}{l}1.27 \\
1.05 \\
1.11 \\
2.25 \\
2.81\end{array}$ & & $\begin{array}{l}1.03 \\
1.00 \\
1.47 \\
3.81 \\
6.93\end{array}$ & \\
\hline
\end{tabular}

Although it would be quite straightforward to insert the coefficients $B_{x}(n, k)$ and $B_{y}(n, k)$ into the computer program, Table 1 suggests that corrections will be small. Also it is to be remembered that the coefficients $a_{n}$ and $b_{n}$ are unknown, that the specified values are rms values, and that the magnets as constructed could conceivably lay outside this range. 
To estimate the contributions of various multipoles, we estimate the terms in Eq. (2.12) as follows:

$$
\begin{gathered}
\left|\left(\hat{x}^{\prime}+i \hat{y}^{\prime}\right)\right| \approx 3.5 \sqrt{2} \sigma_{x^{\prime}}^{*},\left|\left(\tilde{a}_{n}+i \tilde{b}_{n}\right)\right| \approx a_{n} \sqrt{2} 10^{2 n}, \\
\sqrt{\beta^{*} \bar{\beta}_{G}} \approx 40 \mathrm{~m}, \frac{E_{R}}{E_{0}} \approx 0.5 \times 10^{-6}, \quad \sigma_{x^{\prime}}^{*} \approx 10 \mu \mathrm{rad}, \text { and } \sigma_{x^{\prime}}^{*} \approx 5 \mu .
\end{gathered}
$$

Taking out one length factor $\sqrt{\beta_{x}^{*} \bar{\beta}_{G}}$, dividing by $\sigma_{x}^{*}$, and combining that ratio with the leading constant, we arrive at

$$
\left|\frac{\delta x}{\sigma_{x}^{*}}\right| \approx 4.0 \sum\left(\tilde{a}_{n}+i \tilde{b}_{n}\right)\left(\beta^{*} \bar{\beta}_{G}\right)^{n / 2}\left(\hat{x}^{\prime}+i \hat{y}^{\prime}\right)^{n} \bar{g}_{n}^{\beta} .
$$

Upon further insertion of the above estimates, this becomes

$$
\left|\frac{\delta x}{\sigma_{x}^{*}}\right| \approx 4.0 \sum(0.2)^{n} a_{n} \bar{g}_{n}^{\beta} \approx 0.4 \sum(0.2)^{n}
$$

where the product $a_{n} \bar{g}_{n}^{\beta}$ is close to 0.1 for all $n$. The first term in this sum is about equal to 0.016 , and subsequent terms are smaller by the factor 0.2 . The magnitude of the $n=5$ term is $\approx 10^{-4}$, suggesting that this and higher order terms will not be of consequence unless the values of $\hat{x}^{\prime}$ and/or $\hat{y}^{\prime}$ are larger than our estimate.

\subsection{TUNE MOdUlation}

There are many mechanisms that can cause modulation of the betatron tune frequency: power supply ripple, variation of the $\beta$ function with energy, variation of beam-beam interactions resulting from coherent motion, wake field effects, and perhaps others. The specification on the power supplies is intended to guarantee that the tune modulation has an amplitude less than \pm 0.001 . I will study the behavior of particles as the modulation depth and frequency are varied, without reference to the cause of the modulation.

To modulate the tune, it is necessary for each revolution to calculate a sine function. If the initial phase of the modulation is allowed to be different for each particle tracked, then a cosine function is needed as well. Then with magnitude of the tune modulation in hand, the betatron oscillation matrix must be modified. This involves calculating another sine and cosine and multiplying each by two $2 \times 2$ matrices.

Since this is more computation than is preferred at each turn, a large number, $\approx 10^{3}$, of transfer matrices at discrete values of betatron tune are calculated. After the calculation of the particle tune, the transfer matrices nearest in tune are chosen.

This procedure has been criticized on the grounds that it may introduce discontinuities and may of itself give rise to a diffusive process, breaking KAM boundaries. Peggs has 
pointed out that if the phase of the tune modulation were to repeat exactly after an integer number of turns, then the choice of matrices would repeat, and mathematically the result would be equivalent to a machine of circumference this integer number times the design circumference, ${ }^{[5]}$ suggesting KAM boundaries would not be broken. This condition will be satisfied if the tune associated with the betatron tune modulation is a rational number.

The tune itself can be calculated by a $2 \times 2$ matrix multiplication, rather than calculation of a sine and cosine. This uses the fact that a sine and cosine function can be obtained from projecting out components of a vector rotation in a two-dimensional space. The vector rotation matrix in this case is a constant, depending only on the phase advance of the tune modulation after one machine revolution.

\section{FEW-TURN RESULTS}

\subsection{TURN-BY-TURN VARIANCE}

Figure 3 shows turn-by-turn values of variance for the short-range beam-beam force. Tunes are equal to the nominal values, namely: $\left(Q_{x}, Q_{y}\right)=(0.270,0.280)$, and there is no tune modulation. (See Table 2 for values of other input variables.) Only the two low- $\beta$ IRs are included, and the phase difference between the two IPs is held equal to $90^{\circ}$. The variance is evaluated at a point halfway between the IPs.

The variance after one turn gives the mean square value of the beam-beam kick at this amplitude. In a few turns the variance achieves values almost 20 times this size. The maximum value of the variance indicates the extent of deviation of phase space orbits from the initial circle. The periodicity of 50 reflects the fact that $2 Q_{x}$ and $2 Q_{y}$ are rational numbers with common denominator of 50 .

Figure 4 shows turn-by-turn values of the variance for the long-range beam-beam force. All parameters are the same as Figure 3. Note the scale change. Consistent with numerical estimates, the long-range beam-beam force is stronger by a factor of about seven. The character of the turn-by-turn variation is quite different than for the short-range beam-beam force, especially as regards the return to zero.

Figure 5a shows the turn-by-turn values of the variance for the combined short-and long-range beam-beam forces. The short-range beam-beam force slightly modifies the long-range result. The combined forces are used as the base case for the rest of this study. The combination of these forces is calculated by giving a long-range kick, followed by a short-range kick, followed by another long-range kick at each IP.

Also, the turn-by-turn swing in variance in just 20 turns is much greater than the change, $0.01 \in$ in $10^{6}$ turns, we must detect. In the hope of reducing this swing, averages over several turns are examined. 

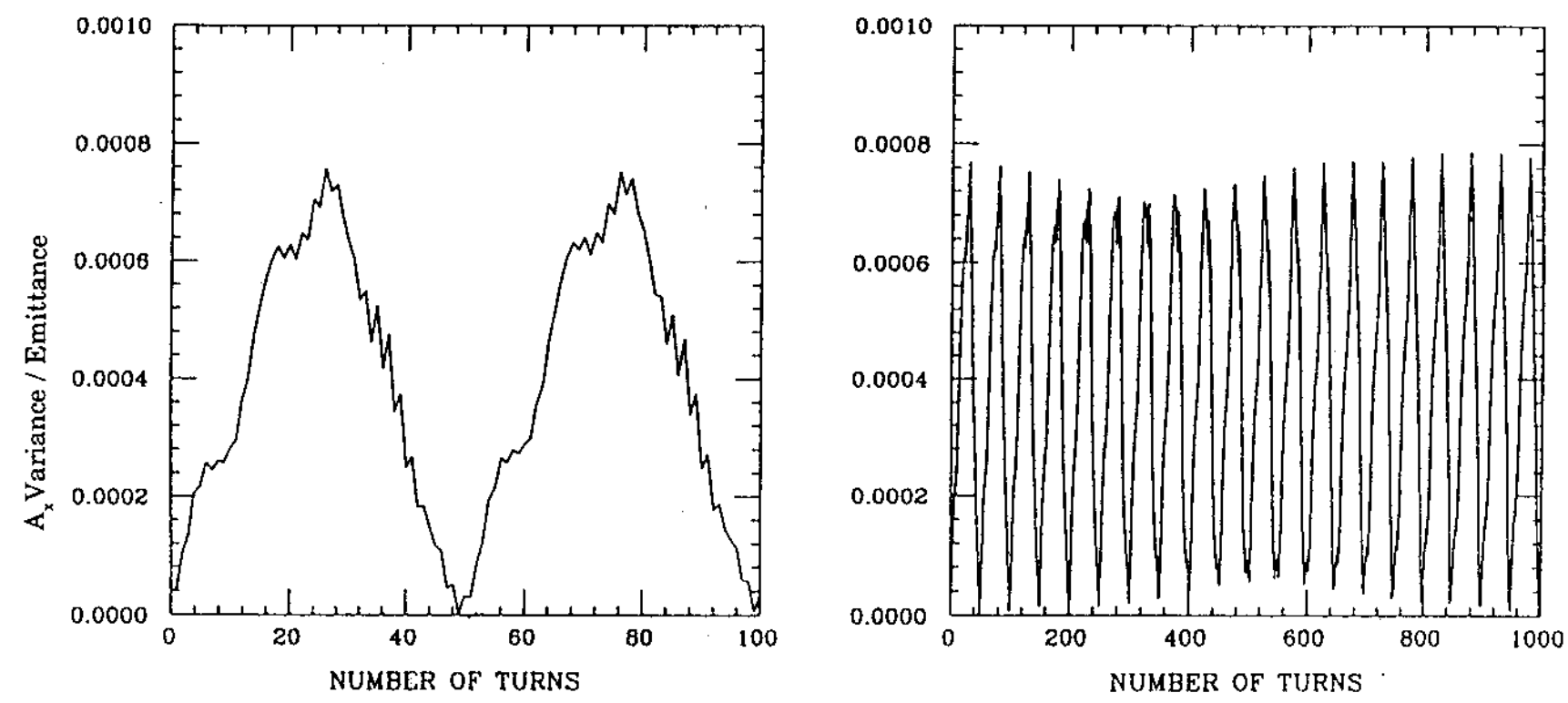

Figure 3. The variance in $A_{x}$, of 100 particles started at $\left(A_{x}, A_{y}\right)=(4.0,4.0)$ with random phases, caused by the short-range beam-beam force.
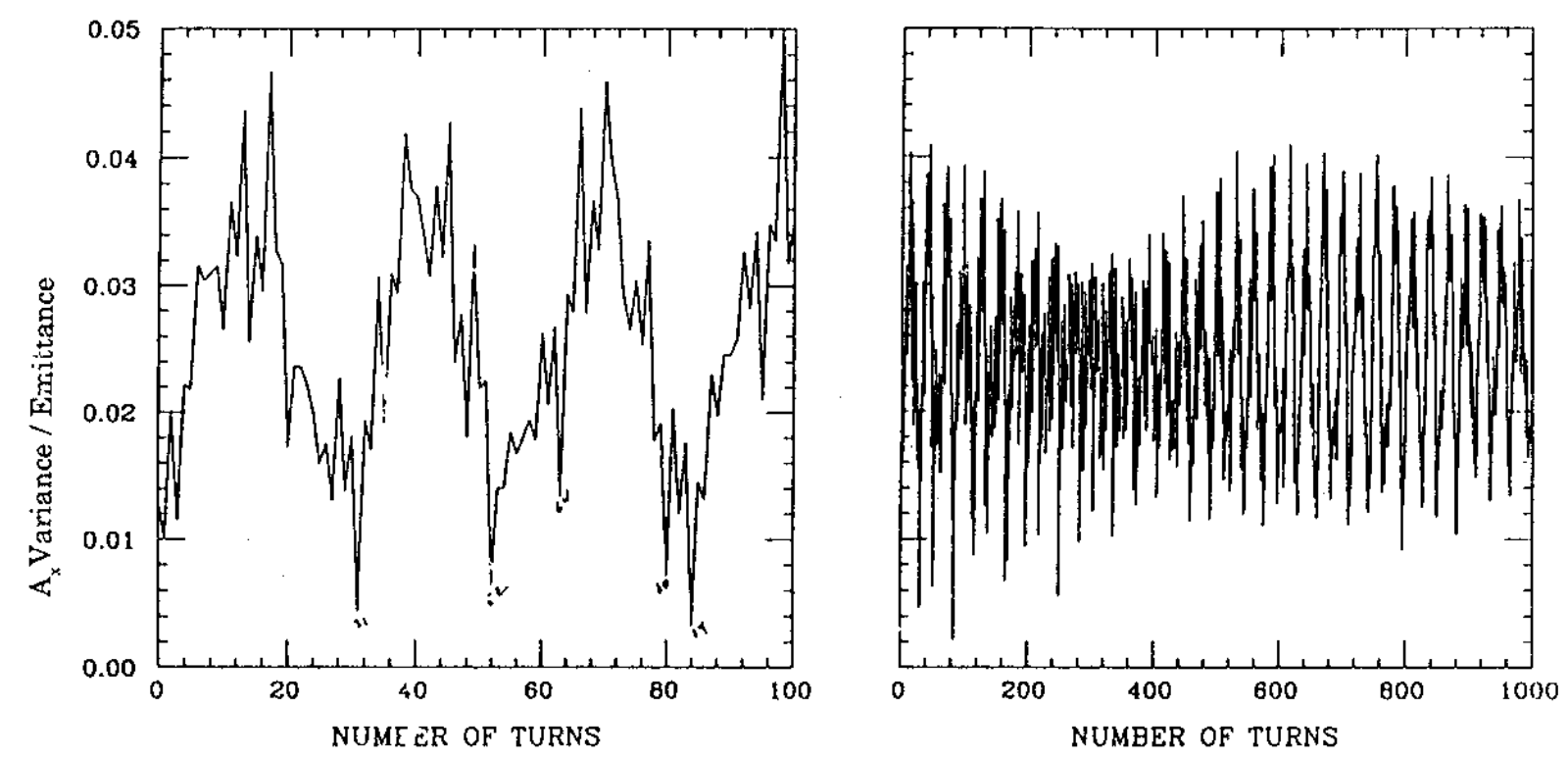

Figure 4. The variance in $A_{x}$, of 100 particles started at $\left(A_{x}, A_{y}\right)=(4.0,4.0)$ with random phases, caused by the long-range beam-beam force. Note that the vertical scale is 50 times that of Fig. 3. 


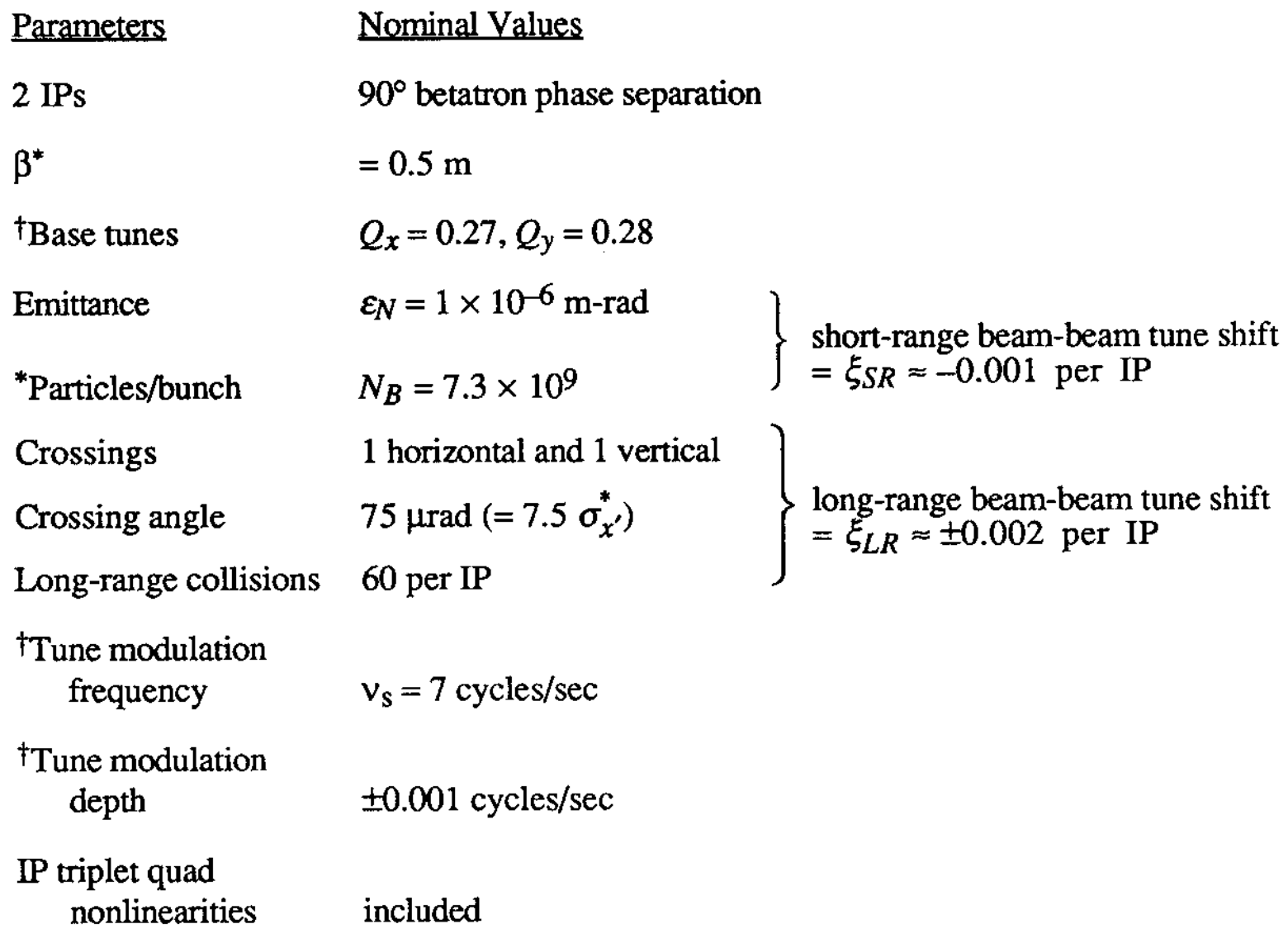

IIndicates that these parameters are varied in this study.

\subsection{MULTI-TURN AVER AGES OF VARIANCE}

Figure 5b shows averages over 100 turns for variances shown in Figure 5a. Turnby-turn averages for more than 400 turns appear to have the required stability; changes in the average value of the variance by $0.01 \epsilon$ should be identifiable. Henceforth, we uniformly average over 1000 turns when calculating values of variance.

For the most part we study initial populations of 100 particles. The fractional accuracy of the sample variance (the "true" variance being the limit of value of the variance as the number of initial particles becomes very large) goes like $1 / \sqrt{N}$, where $N$ is the number of particles in the sample. $N=100$ should therefore provide 10 percent accuracy. Since the initial value of the variance is of minor importance, compared to the growth rate of the variance, 10 percent accuracy seems adequate. 

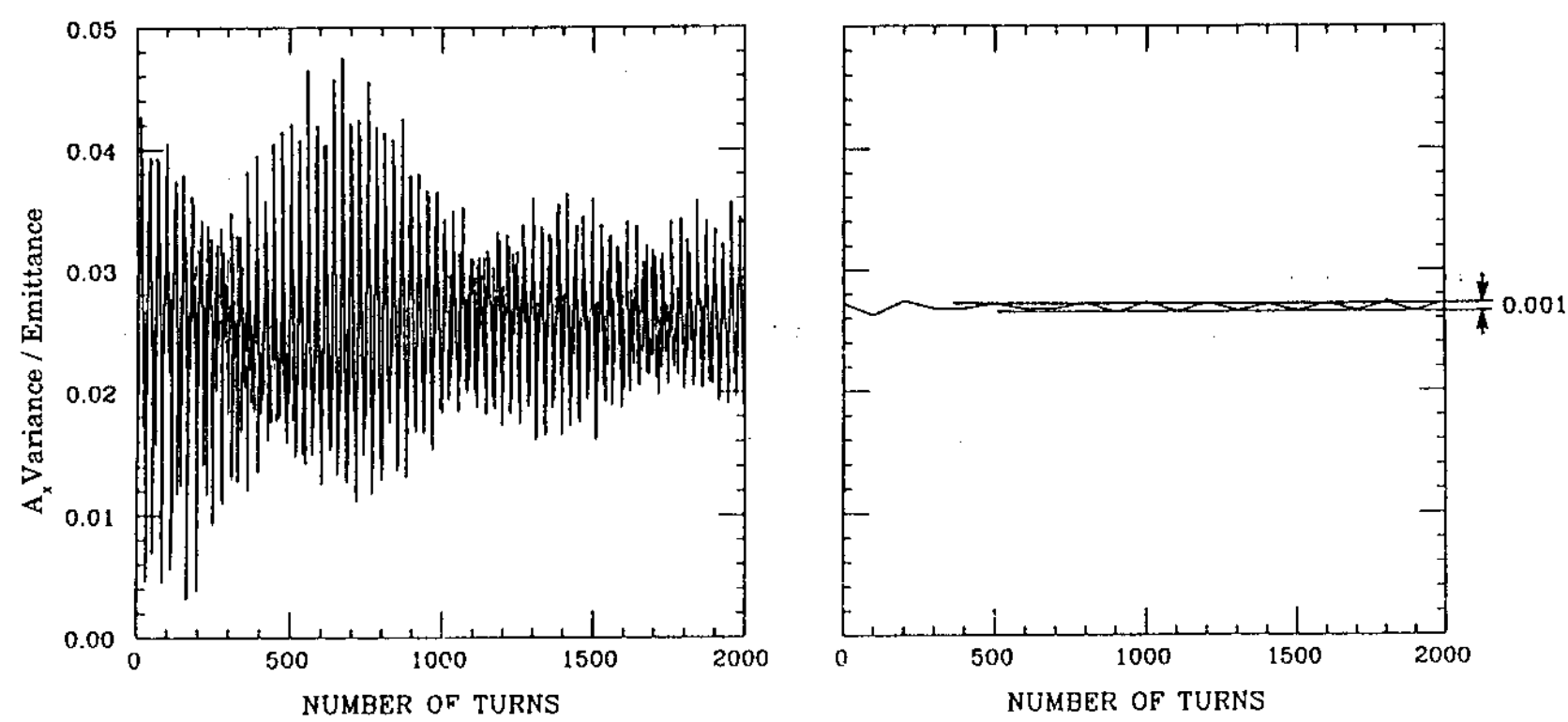

Figure 5. The variance in $A_{x}$, of 100 particles started at $\left(A_{x}, A_{y}\right)=(4.0,4.0)$ with random phases, caused by the combined short- and long-range beam-beam force. Figure $5 \mathrm{~b}$ is the same data as Fig. 5a, averaged over 100 turns. The average is already stable to within our desired sensitivity of \pm 0.001 .

However, one might argue that it would be preferable to study a population of ten particles or even one particle, since the growth in variance increases linearly with the number of turns, while the accuracy increase only as the square root of the number of particles. For the same number of particle turns, it appears advantageous to maximize the number of turns.

If computer time were unavailable to study a sample, I would concur: tracking one particle has the best chance of revealing diffusive action. But this leads to a concern that the initial coordinates might be special in some way. Also it may be that the diffusion model is naive and that initial conditions are more important than such a model would suggest. Then one would wish to consider as many initial conditions as possible.

I have decided to take the point of view that this is a "statistical" study: that one should have a good sample of initial conditions, and through the course of the work establish the accuracy of the diffusive model. Also, when starting with samples of ten particles it is often difficult to compare results, as one or more of the variables were changed. This led to a sample of 100 particles. It should be mentioned that compilers now take advantage of loop structures, so that multi-particle calculations are more efficient. On the CRAY, 64 particles may be tracked almost as quickly as one particle. 


\subsection{TUNE Plane CONS IDERATIONS}

In Figure 6 we show that the variation of the initial amplitude, averaged over the first 1000 turns, increases with amplitude as expected. The initial variance also varies dramatically with tune. This is shown in Figure 7, which shows the dependence of the initial variance on the $Q_{y}$ tune for four different values of $Q_{x}$ tune.

To understand Figure 7 we look at the resonances encountered in this tune plane region. Figure 8 shows the entire tune plane with all resonances of order less than or equal to ten. On this diagram, the box outline includes the tune region of interest, and this region is shown in Figure 9. It is now easy to identify the "resonant-like" features of Figure 7. The variance increases occur as we encounter the low-order resonance lines. Of course, there is a tune shift that depends on amplitude due to the short- and long-range beam-beam force, and the features shown in Figure 7 will be slightly shifted from the position of the lines in Figure 9.

As a result of several such studies of initial variance, for our base operating point for the rest of these studies we have chosen a region as far from resonant features as practical. This point, shown on the lower left-hand graph of Figure 7, is $\left(Q_{x}=0.27, Q_{y}=0.28\right)$. Figure 9 shows the range of tunes with amplitude that we expect to encounter as we vary the amplitude. We see that the operating region is quite devoid of low-order resonances.

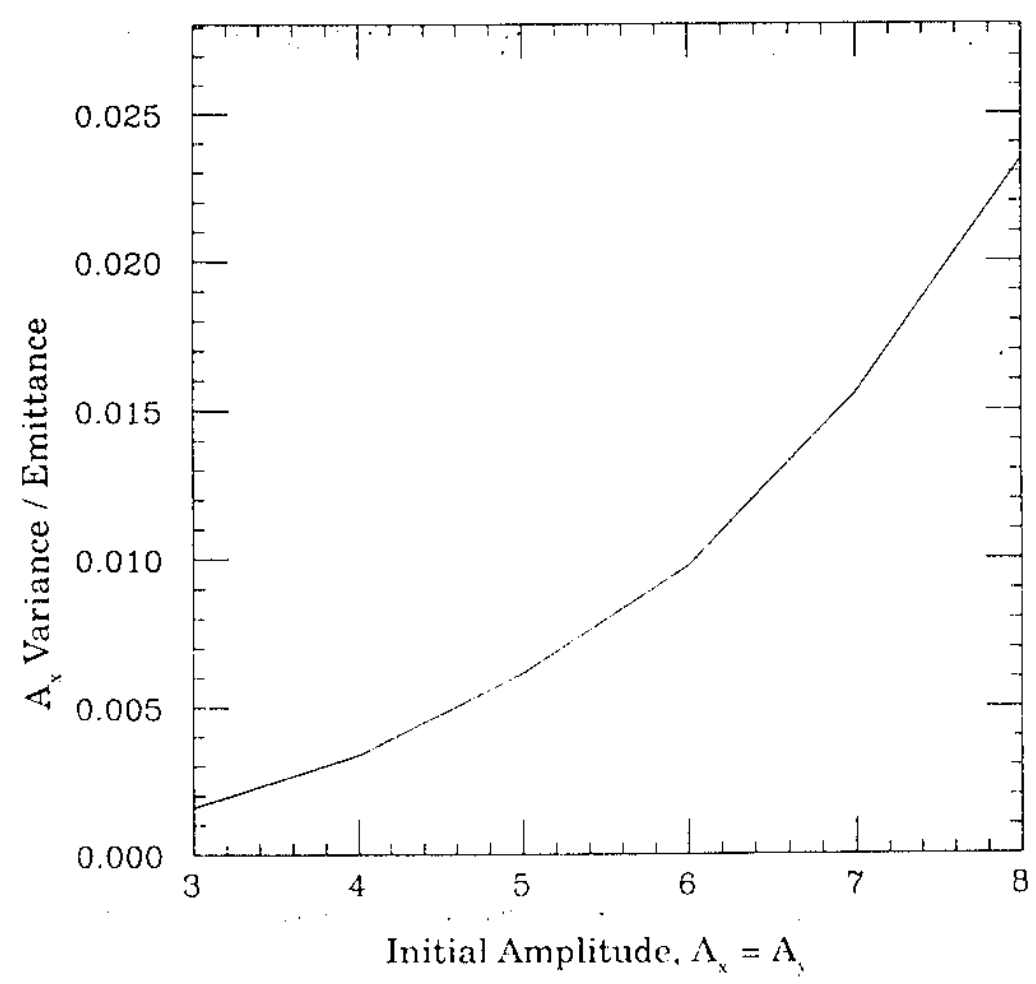

Figure 6. The initial variance (averaged over the first 1000 turns) depends upon the starting amplitude. 


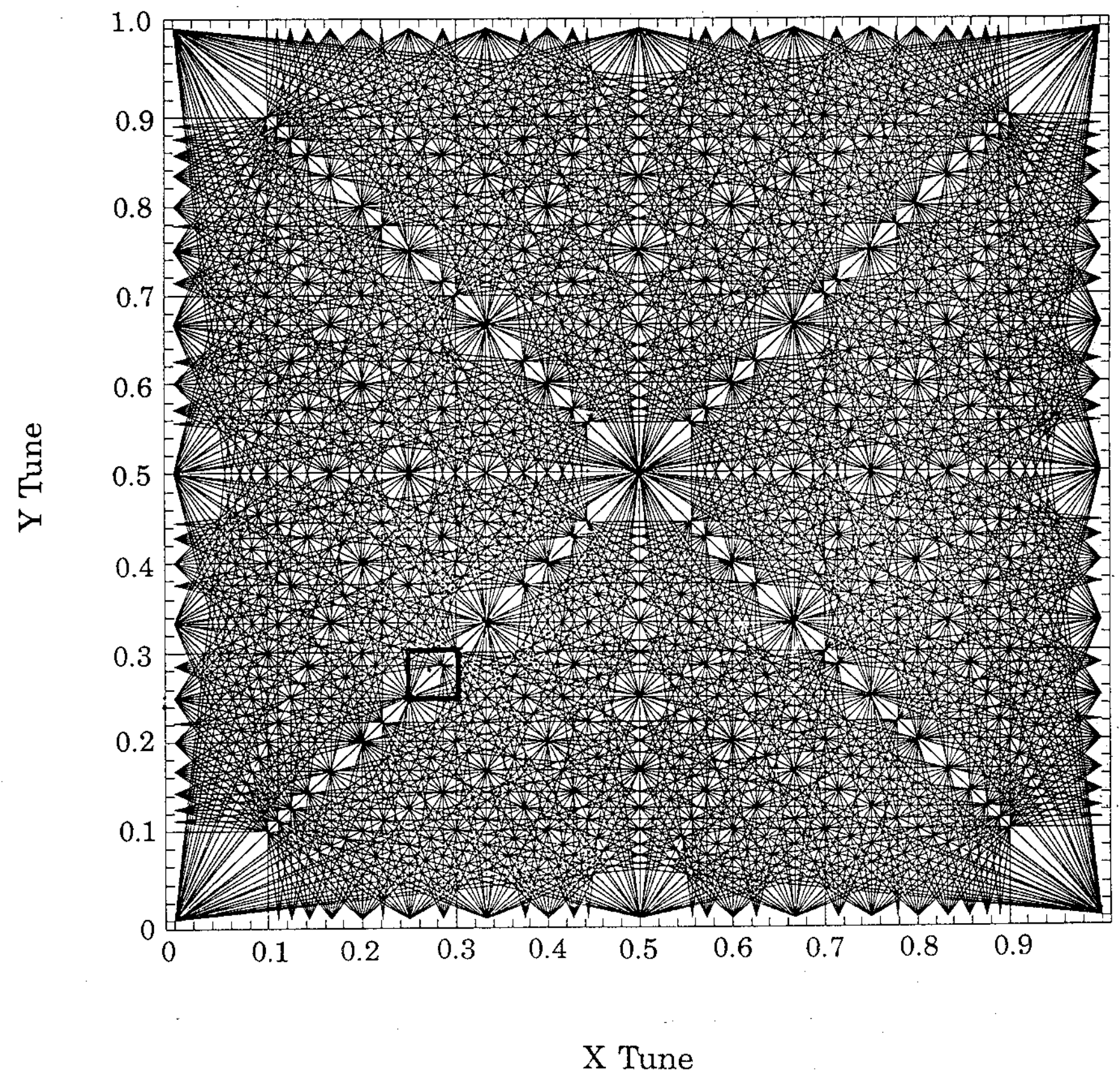

Figure 7. The tune plane showing all resonances of order less than or equal to 10. (Taken from Tennyson, SSC-155[4]) The heavily outlined box is the region studied in this report. 

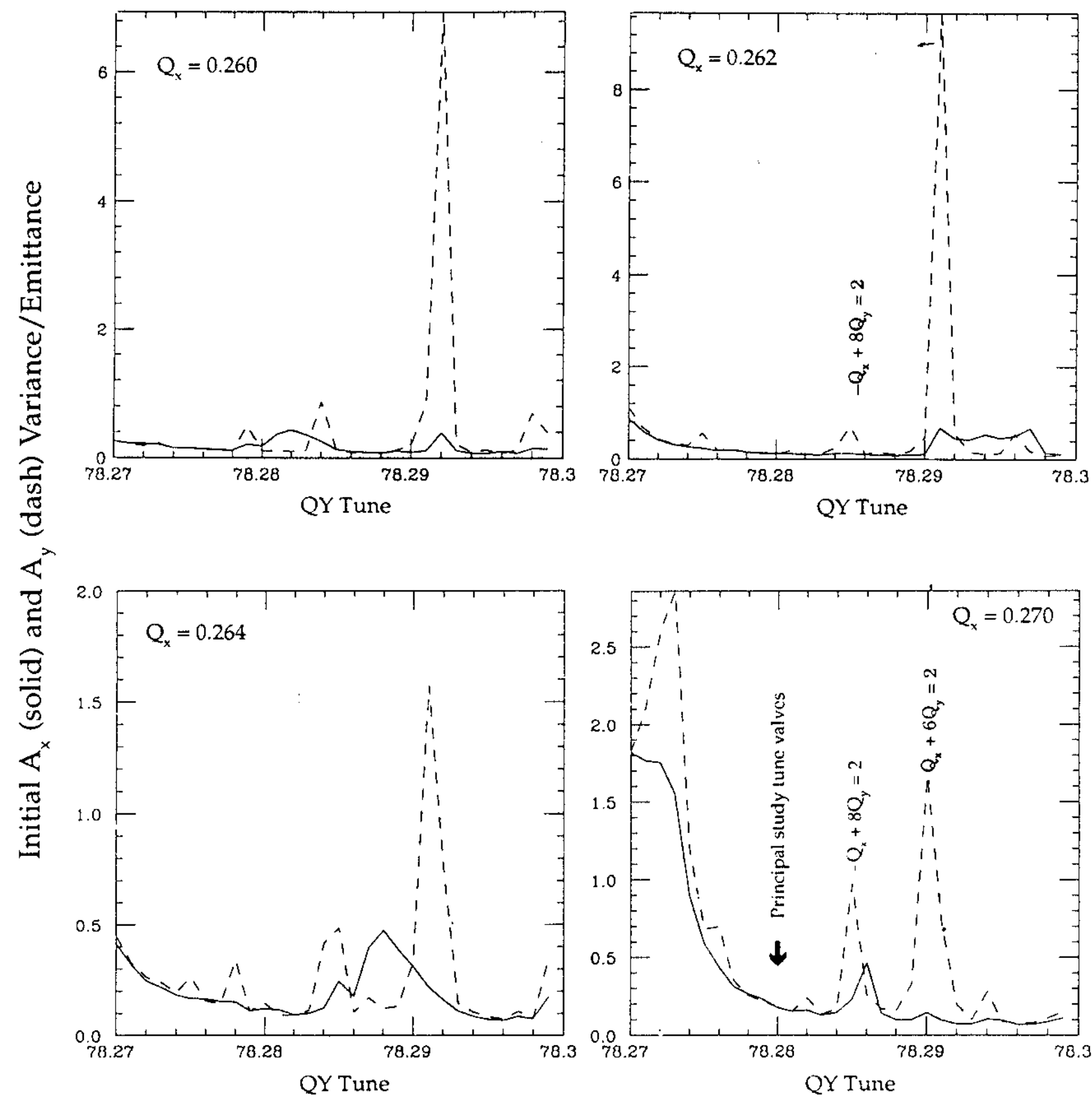

Figure 8. The initial $A_{x}$ and $A_{y}$ variance depends strongly on the base tune. By taking cuts at several $Q_{x}$ tunes it is possible to identify important resonance lines. 


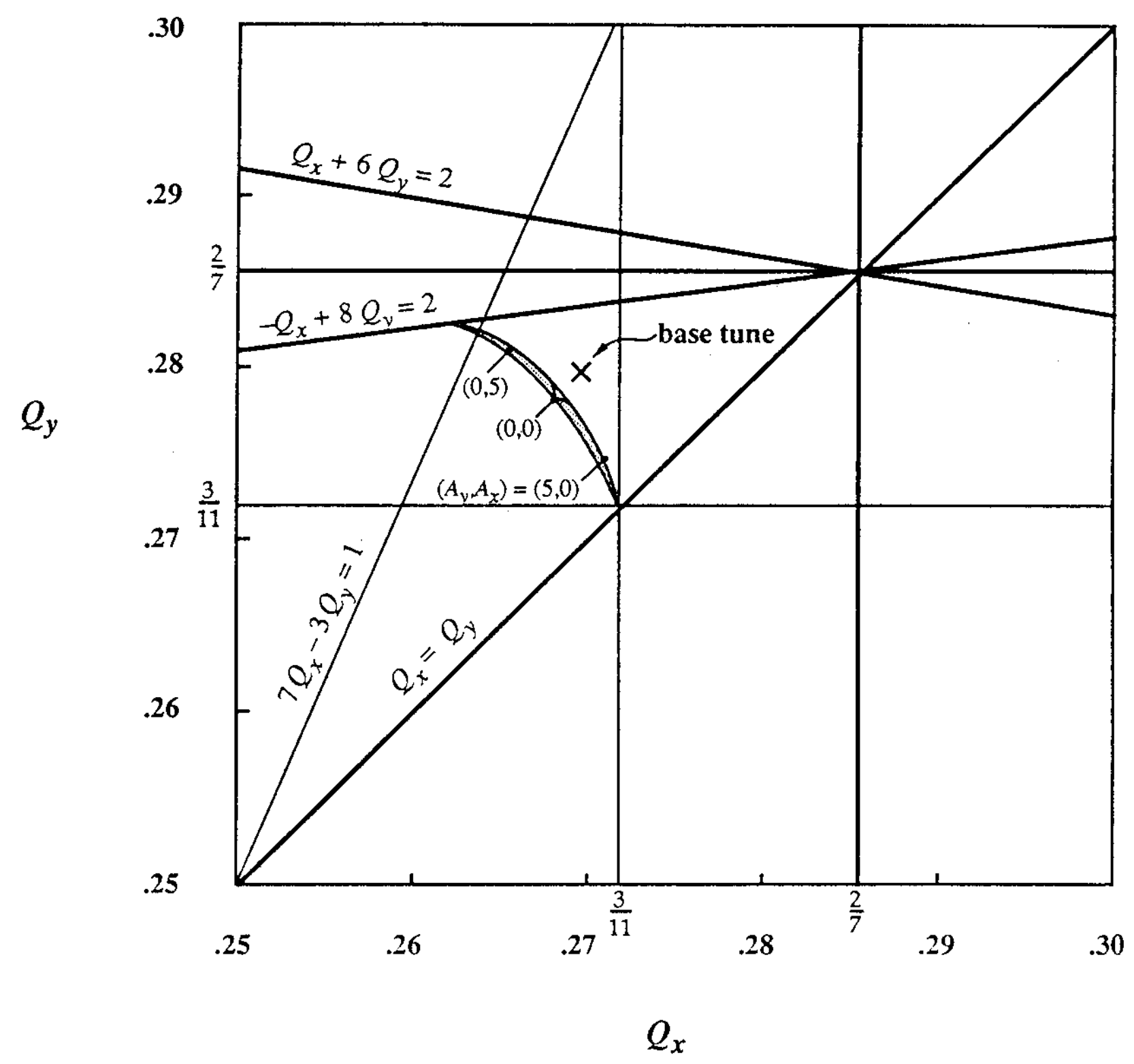

The Tune Plane

Figure 9. The tune plane for $(0.25,0.25) \leq\left(Q_{x}, Q_{y}\right) \leq(0.30,0.30)$, showing important beam-beam resonances and the base tune chosen for the studies of tune modulation. The shaded region indicates the theoretical tune shift with amplitude (see Figure 11, Tennyson, SSC-155[4]). 


\section{MANY-TURN RESULTS}

\subsection{Diffusive BOUNDARY With AND Without TUNE MOdUlation}

Now begins the search for the presence of diffusion, at first without tune modulation. Figure 10 shows the $A_{x}$ variance, averaged always over 1000 turns, for four starting amplitudes, for $10^{6}$ turns. Note that the scales on the four graphs are each different. At $A_{x}$ $=A_{y}=6.0$ we begin to note a wandering of the variance of an amount equal to our cutoff criteria of 0.01 . However, the distinctive signs of diffusion for which we are looking are not evident. At $A_{x}=A_{y}=7.0$ there is a significant and unmistakable growth in the variance. These graphs are typical, and illustrate that without tune modulation we find evidence for an onset of diffusion at amplitudes comparable to the amplitude of the beam separation, about $7.5 \sigma$.

Contrast this picture to the variance in Figure 11 when tune modulation is present; the conditions are those described in Table 2. At $A_{x}=A_{y}=3.0$ the motion appears to be stable; at $A_{x}=A_{y}=4.0$ there is clear evidence of diffusion. Thus I conclude that modulation effects the diffusive dynamic aperture in an important way. Modulation is in practice unavoidable, and so it represents an important part of the physics we must consider when trying to identify the long-term dynamic aperture.

In Figure 12 a diagram of the amplitude plane summarizes the exploration of the diffusive boundary for the conditions described in Table 2 . The dots indicate the points in amplitude plane for which we have tracked 100 particles for $10^{5}$ or $10^{6}$ turns. Results typical of this tracking are displayed in Figure 13. The first graph here shows no signs of variance growth; the second graph shows a growth of $0.001 \epsilon$ and indicates the presence of some diffusion; the third graph indicates marked growth in variance.

In looking at such graphs one can make the following observations: (1) The DDA doesn't depend strongly on the exact definition chosen for the magnitude of the diffusion. The onset of diffusion is quite noticeable and abrupt. Uncertainties are the order of $0.2 \sigma$. (2) There are amplitudes for which the behavior of the variance is unclear. It meanders and oscillates, and it is unclear whether motion at that amplitude will be stable in the long term or not. Fortunately, such ambiguous behavior is limited to a small range of amplitudes, again the order of $0.2 \sigma$. (3) Occasionally one encounters a region of observable diffusion, and then at larger amplitudes diffusion is not present. For this reason we have defined the DDA as the largest amplitude with no observable diffusion at smaller amplitudes. The "holes" are not widespread or of particular significance in our judgement. 

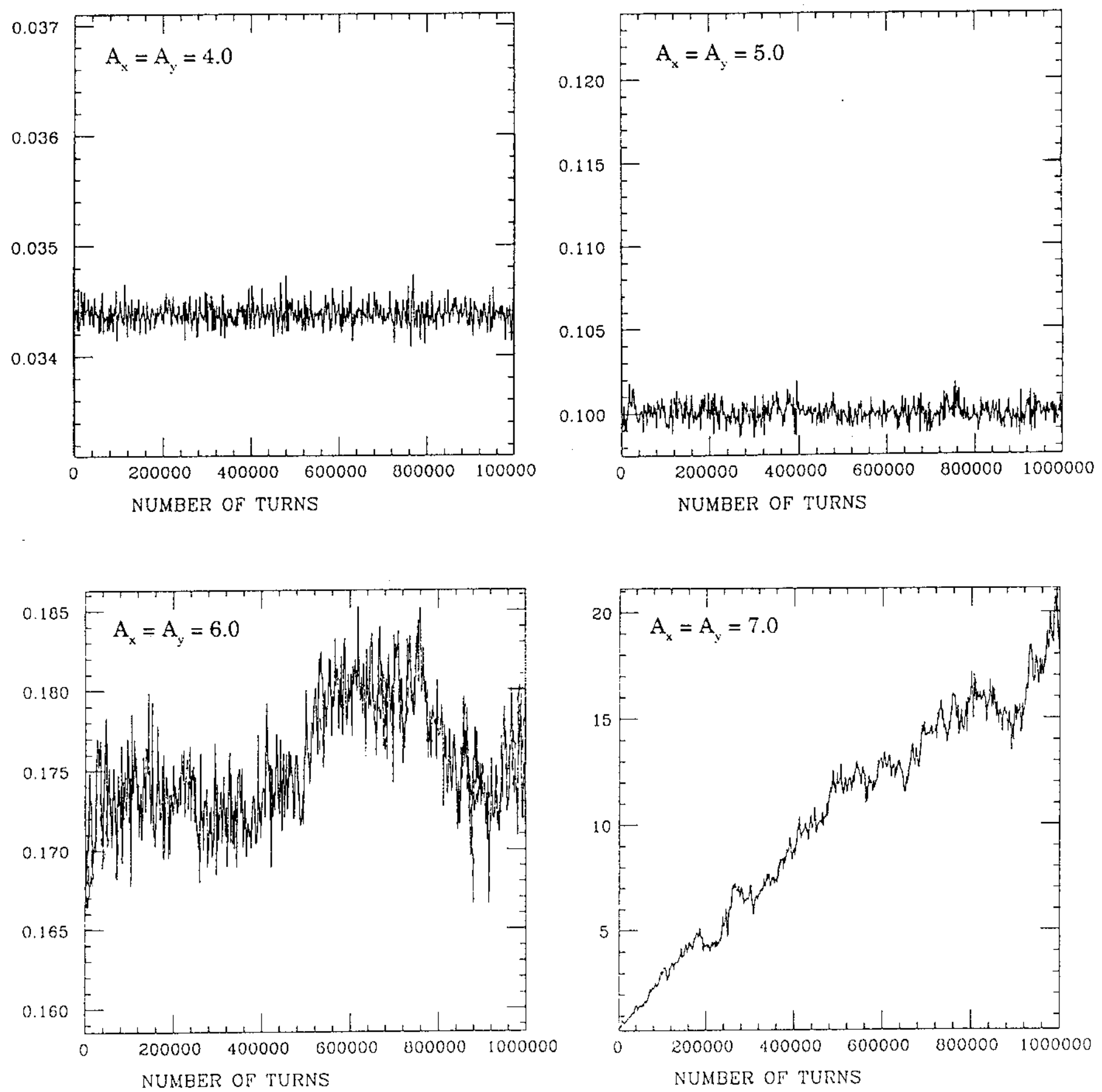

Figure 10. The " 1000 -turn averaged" $A_{x}$ variance without modulation, for several starting amplitudes. At $A_{x}=A_{y}=4.0$ and 5.0 the variance seems quite stable, at 6.0 it oscillates and meanders, and at 7.0 there is significant indication of diffusion. 

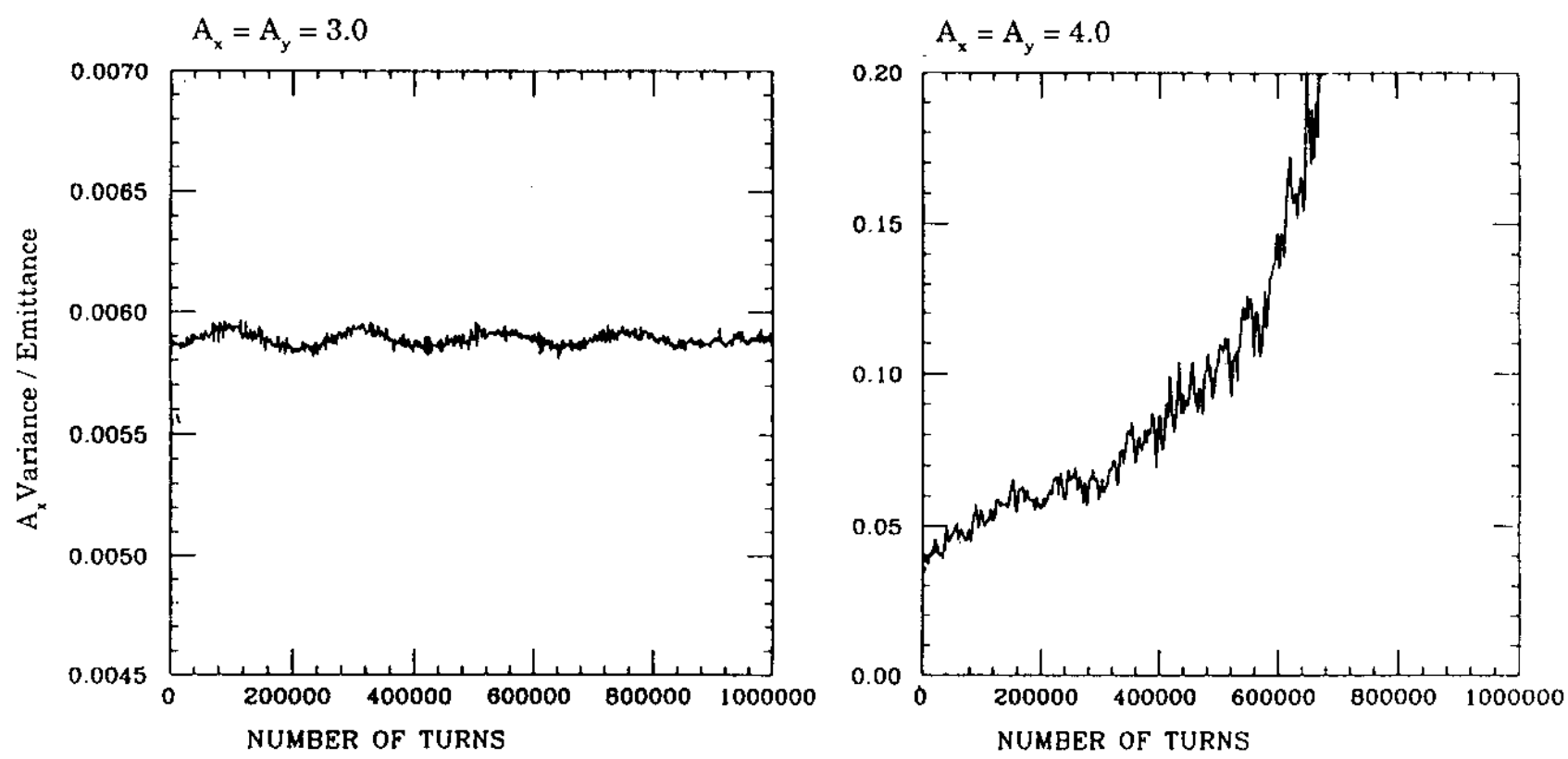

Figure 11. The "1000-turn averaged" $A_{x}$ variance with tune modulation included. Now there is indication of diffusion at $A_{x}=A_{y}=4.0$.

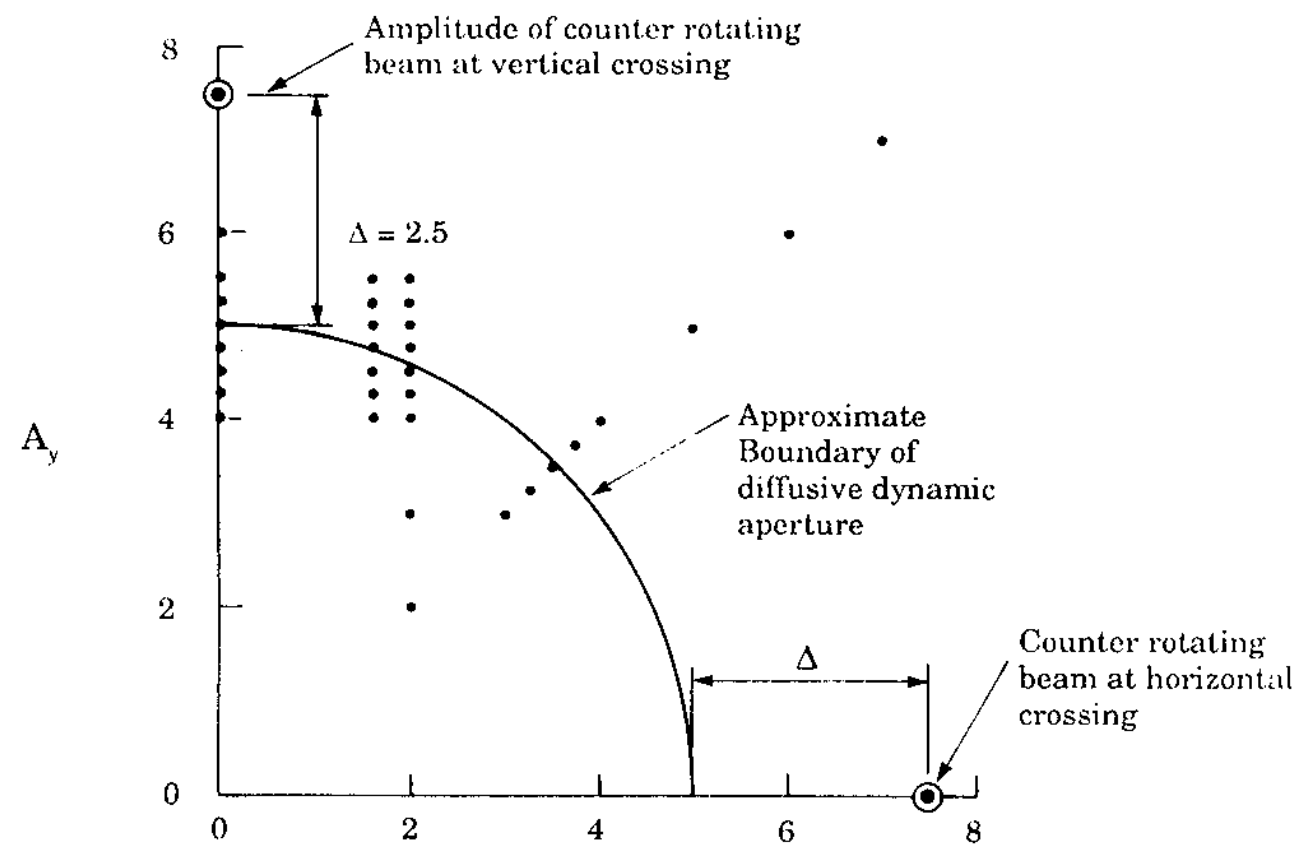

$A_{x}$ [units of $\sqrt{\varepsilon}$ ]

Figure 12. The amplitude plane. The dots show the points explored in this study. The circles indicate the location in amplitude space of the counter-rotating beam at the two crossings. 

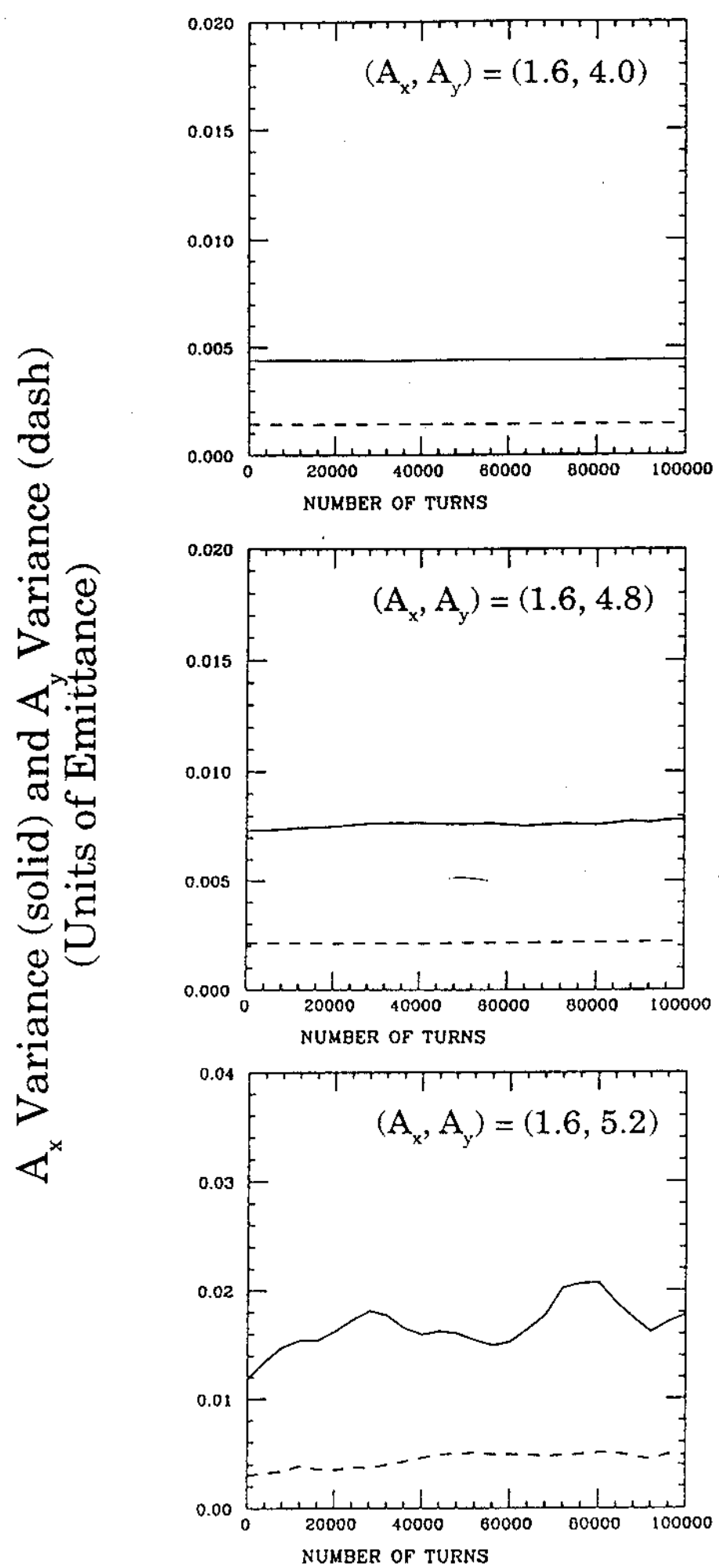

Figure 13. A typical set of graphs used to determine the location of the diffusive dynamic aperture. In the first graph, there is no indication of diffusion, in the second there is a graph of the variance of less than 0.001 in $10^{5}$ turns; hence $\left(A_{x}, A_{y}\right)=(1.6,4.8)$ lies outside the diffusive dynamic aperture. The last graph gives clear indication of increasing variance. 


\subsection{Change in Diffusive Boundaries as Parameters ARE VARIED}

The DDA for various modulation depths and modulation frequencies were determined. Modulation depths of $\pm 0.00025,0.0005$, and 0.001 were used. Over this range of modulation depths no significant change was observed in the position of the DDA. In other words, even quite small modulation depths are of profound significance for the determination of the long-term behavior.

The DDA did change with the modulation frequency. These results are presented in Figure 14. It is easiest to present the result in terms of the parameter $\Delta$, which is defined in Figure 12 . We find the DDA has a minimum ( $\Delta$ has a maximum) at a frequency of about two cycles per second (about 2100 turns per modulation cycle).

Finally I studied the behavior of the DDA when the crossing angle and the beam intensity were varied. This is of especial interest since it is contemplated that the beam intensity may be increased at some later time to achieve a larger luminosity. In Figure 15 the parameter $\Delta$ is shown to be constant over a wide range of crossing angles. I found this quite remarkable and regret the lack of time to study this further. It would appear to be a strong lead toward identifying the mechanism that is responsible for the onset of diffusion. This simple result inspired me to simplify the long-range beam-beam force, retaining only the $1 / r$ behavior (omitting the changes in the force that occur as a particle probes the interior of the counter-rotating charge distribution). The DDA was unaffected by this simplification.

It follows from the above paragraph, and a simple scaling argument, that the parameter $\Delta$ should vary as the square root of the beam intensity. I studied this parameter change, and the result is presented in Figure 15. The parameter $\Delta$ does indeed follow a square root curve. 


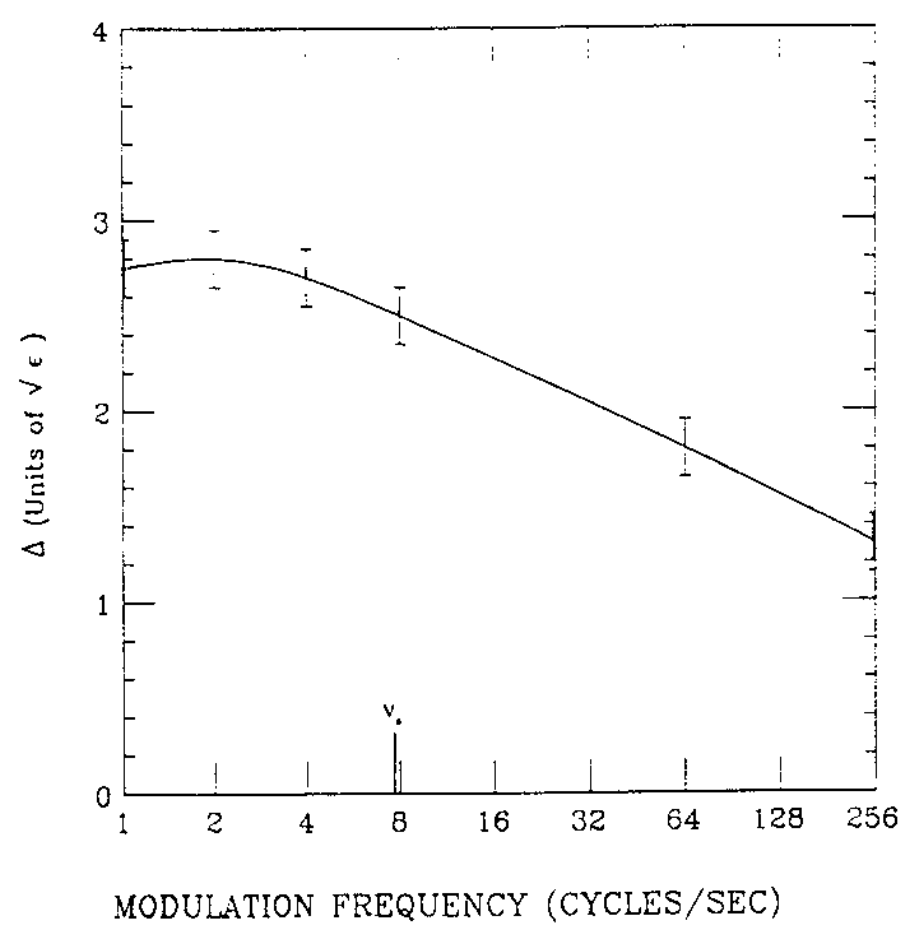

Figure 14. The diffusive dynamic aperture varies with modulation frequency (see Fig. 12 for the definition of $\Delta$ ).

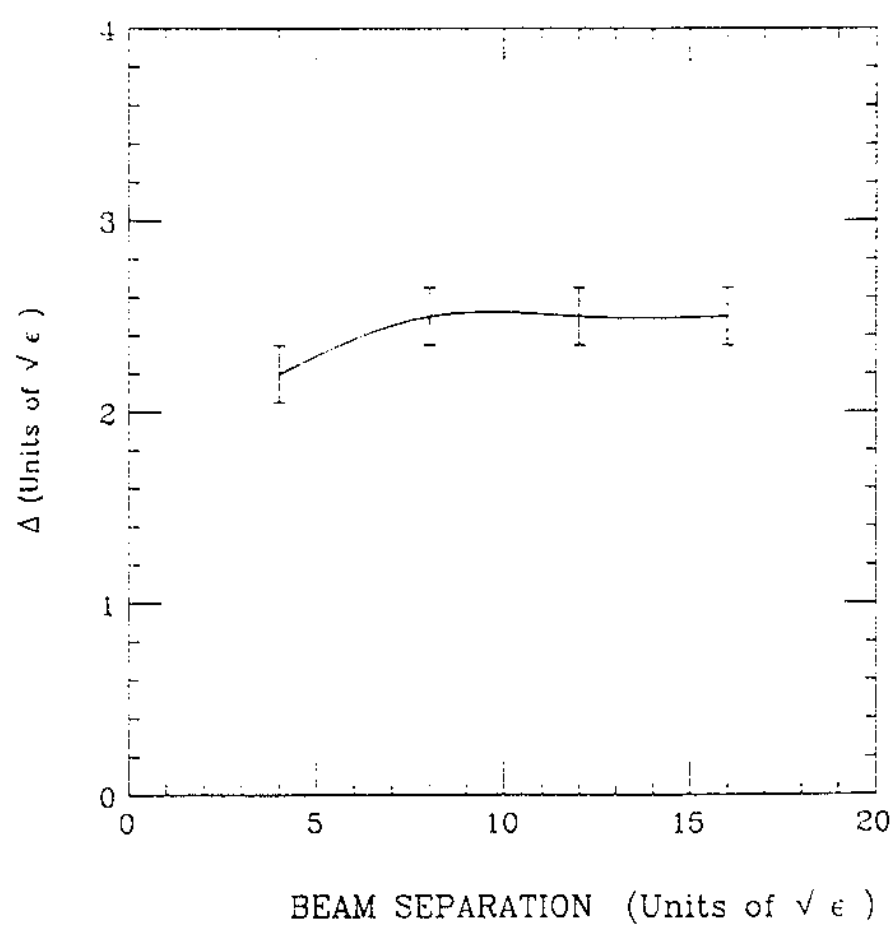

Figure 15 . The parameter $\Delta$, determining the diffusive dynamic aperture, is nearly independent of beam separation, over a wide range of beam separations. 


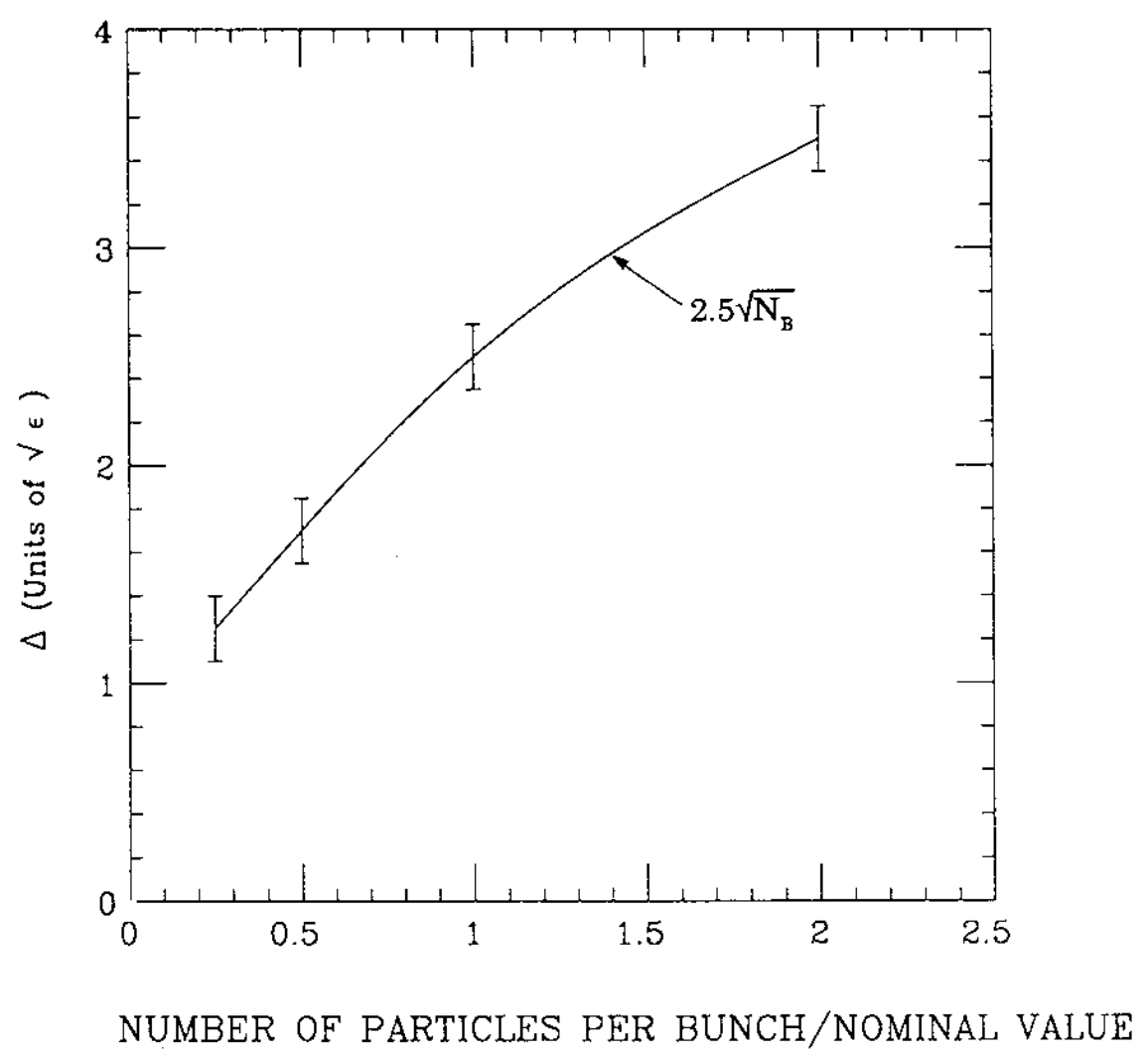

Figure 16. The parameter $\Delta$ varies as the square root of the beam intensity.

\section{CONCLUSIONS}

Actual tracking indicates that particle loss is more complex than would be indicated by a pure diffusion model, but that a diffusive dynamic aperture (DDA) as presented can be defined and can be a useful concept. In particular: (1) the DDA is quite insensitive to the actual specification on growth rate chosen, varying by less than $0.2 \sigma$ for any reasonable choice for the growth rate, (2) the DDA without tune modulation is about equal to the longrange beam separation, (3) the DDA decreases by about $2.5 \sigma$ when the tune is modulated at the synchrotron frequency (about 7 cycles per second), (4) it becomes larger with increasing frequency and smaller by about $0.3 \sigma$ with decreasing frequency (the smallest DDA appears to occur at about 2 cycles per second), (5) it varies only slightly with modulation depths greater than 0.00025 cycles per second (up to 0.001 ), (6) this tunemodulated boundary is purely a property of the $1 / r$ force between bunches, it is not determined by "particles beginning to pass through the other bunch," (7) it is not altered significantly by inclusion of the short-range beam-beam force, nor the nonlinear kicks of the triplet quadrupole, and (8) it does depend strongly on the current. The aperture reduction of $2.5 \sigma$ quoted in item (3) varies as the square root of the current. 


\section{ACKNOWLEDGEMENTS}

The author would like to acknowledge many fruitful conversations with Steve Peggs, Jeff Tennyson, and Jack Peterson; to thank Tjet Sun, Miguel Furman, and Ruth Hinkins for help with the LBL and MFE computers; and to express deep gratitude to Alex Chao for inviting him to join the SSC Central Design Group to work on this problem.

\section{REFERENCES}

1 Conceptual Design of the Superconducting Super Collider, edited by J. D. Jackson, SSC Central Design Group, SSC-SR-2020 (March 1986), p. 95.

${ }^{2}$ M. Furman and A. Chao, "Effect of Long Range Beam-Beam Interactions in the Stability of Coherent Dipole Motion, SSC Central Design Group," SSC-28 (April 1985). M. Furman, "Results of Coherent Dipole Beam-Beam Interaction Studies of SSC Lattices," SSC-62 (May 1986). E. Forest, "A Theory of Coherent Beam-Beam Effects with Long Range Interactions," SSC-67 (March 1986).

${ }^{3}$ Marx, J. and R. Donaldson, eds. Proceedings of the 1986 Summer Study on the Physics of the Superconducting Super Collider, SSC Central Design Group, p. 547 (June-July 1986).

4 J. Tennyson, "Beam-Beam Stability in the SSC," SSC-155, SSC Central Design Group (January 1988).

5 A. Chao, "Errors and Corrections," SSC-N-440, SSC Central Design Group (January 1988).

6 S. Peggs, private communication, August 1987. 